\title{
COMMUNICATION AND PROCESS SIMULATION OF SET-BASED DESIGN FOR CONCRETE REINFORCEMENT
}

\author{
John-Michael Wong \\ Kristen Parrish \\ Iris D. Tommelein \\ Bozidar Stojadinovic \\ Department of Civil and Environmental Engineering \\ University of California, Berkeley \\ Berkeley, CA 94720-1710, U.S.A.
}

\begin{abstract}
Two discrete-event simulations are developed to assess the feasibility of improving the delivery process of reinforced concrete structures. The simulations represent the resource and information flows necessary for supporting set-based design of reinforcing bars used in concrete. Setbased design enables the exploration of feasible solutions for longer in the design process than is otherwise affordable using point-based design, so as to allow for input from several project participants early on and at the same time. The simulations use the canonical example of selecting longitudinal reinforcement for a beam-column joint. The first simulation uses EZStrobe $(0$ to model the amount of time necessary to converge to a satisfactory design solution using set-based vs. point-based design methods. The second simulation uses XML data exchange and state machines in a service-based architecture to analyze the required information flows for communicating design sets. This enables the exploration of a metalanguage to express design goals.
\end{abstract}

\section{INTRODUCTION}

Reinforced concrete is used in capital facilities in all sectors of the construction industry. Specialists involved in its supply chain include owners, architects, structural engineers, steel mills, concrete suppliers, reinforcing bar fabricators, placement contractors and their craft laborers, general contractors, formwork contractors, and others. That they are numerous reflects the advanced technological understanding and capabilities that we exploit today to build structures to meet increasingly stringent owner and societal requirements. The need to specialize, however, has gone hand-in-hand with industry fragmentation resulting in a sub optimal project delivery process. Metrics of performance leave no doubt that current practice can be improved significantly: there are many unnecessary iterations and large amounts of rework in design and detailing, numerous requests for information (RFI) from the builder to the designer, long lead times to fabricate and deliver rebar, time consuming and costly constructability problems discovered during on-site placement, tons of paperwork cluttering the process, and huge legal issues challenging many a project.

To globally improve project delivery, participants from across the supply chain must collaborate starting at the project outset to exploit the unique process and product capabilities of individual members of the team as well as synergistic and collaborative relationships that may be developed within the team. Furthermore, early collaboration may be supported using set-based design, a methodology that encourages all participants to engage in exploring the entire design space. This design space is narrowed collectively until a globally optimal design is found.

Simulation of set-based design is presented using an example of designing longitudinal reinforcement for a concrete beam-column joint.

\subsection{Point-Based Design}

A point-based design methodology is often used in structural engineering. Point-based design involves selecting a single structurally-feasible design option early and then refining that single design (or point) as more information becomes available throughout the design process. This single design is then re-worked until a solution is found that is feasible for all parties. The first design selected tends to be uninformed by the expertise of rebar fabricators, placers, and concrete suppliers who will perform the actual rebar detailing, rebar placement, and concrete placement. Not including their expertise tends to produce a design that may be optimal for structural performance yet suboptimal from an entire project perspective. This is 
not to imply that the structural engineer is not concerned with constructability. Rather, local optimality is a byproduct of the structural engineer being hired to develop one point solution. Structural engineers design for constructability to the extent they are able to. However, considerations pertaining to rebar fabricator capabilities are not necessarily considered. Likewise, contractors may offer their expertise, but the time and contract structure used in most design projects does not allow for this conversation to significantly impact the initial design. Clearly, there is much room for improvement in point-based design.

\subsection{Set-Based Design}

In contrast to point-based design, set-based design focuses on keeping the design space as open as possible as long as possible. The design space is articulated differently in different companies, depending on use. For instance, in automobile manufacturing, Toyota spends a lot of time upfront doing experimentation to fully explore the design space (Ward et al. 1995). In structural engineering, the design space can be defined as a collection of specific design options. Dependent upon the necessary level of abstraction, the sets can be continuous or enumerated as discrete design options. Specific designs are not considered alone; rather, the set of options that remain in the design space are considered feasible. Instead of specifying a design before all the constraints are known, design decisions are postponed until the "last responsible moment". Ballard (2000) defines the last responsible moment as the "point at which failing to make the decision eliminates an alternative".

Set-based design is analogous to a funneling process. At the start, there are many design options. As constraints are invoked, the number of designs still feasible is reduced (Sobek et al. 1999). In order to implement setbased design, there must be a work structuring effort to determine who the stakeholders are as the project unfolds. It is important to recognize that the relevant stakeholders will likely be different at various phases of the project. A key to the success of set-based design is knowledge sharing; whenever the feasible design space is reduced, the reason for eliminating any part of the feasible design space needs to be documented and made accessible to all relevant stakeholders. Preserving the maximum number of feasible designs as long as possible reduces the likelihood that rework will be necessary and allows all project participants to utilize their unique expertise to make the project successful.

\subsection{Simulation for Set-Based Process and Information}

Simulation models are presented here to help assess the use of set-based design methods for reinforced concrete structures. A process-based and information-based simulation are developed to represent the resource and information flows necessary for point-based and set-based design. EZStrobe $(\mathrm{C}$ is used to model the overall process. Java web services and browser-based access are used to model the communication and process implementation.

A process-based simulation allows for the exploration of resources and coordination points necessary within the design methodology. Communications, in the form of approvals and transmittals between the project participants, are modeled as discrete activities that have a specified time duration. This type of simulation is well-suited for models with a high level of abstraction and for investigating how cross-functional teams operate in a set-based environment.

An information-based simulation documents the information required for transition from a point-based to a set-based design methodology. Since sets must be communicated differently than points in the design space, more data exchange is required among the project team. A web services-based architecture for the simulation allows the structural engineer (SE), general contractor (GC), and rebar fabricator (RF) to communicate collaboratively while still remaining as distinct entities within the model. $\mathrm{XML}$ is used as the data exchange language to allow future expansion and standards-based communication. This information can be used to enable knowledge sharing across the project team.

The cognitive processes and language/action cycle associated with the flow of design information are not modeled at this time.

\section{RELATED WORK}

Many references describe the usefulness of simulation for improving the current state of engineering and construction practice. Halpin and Martinez (1999) investigate factors that contribute to successful simulation implementation in construction firms. Their use of the CYCLONE methodology for simulating operations that are cyclical or repetitive achieved a substantial performance increase with a floating caisson example project.

Kamat and Martinez $(2000,2001)$ identified that lack of visualization is a barrier to using simulation data for decision making. To address this, a 3D visualization system was developed that complements discrete-event simulation results. The ability to visualize the simulation allows its users to develop a deeper trust and understanding of the model. 
Service-based systems for structural design have been implemented by other researchers, however relatively little information exists in the literature regarding a system that explicitly involves set-based design. PenaMora and Wang (1998) presents a method for bidding negotiation using a computer tool. Pena-Mora and Choudri (2001) show details for the information necessary for making legally binding electronic decisions. Anwar et al. (2005) use the notion of an "information bus" to accommodate data interchange between project participants during different project phases. Component-based frameworks are developed to translate common structural information to the specific formats needed by each party using XML with XSLT. Project phases are modeled as "packages" that take inputs from the information bus and output more information to the bus. Anwar provides an example of how to formalize communication for the authoring of computer tools and software. This model assumes that all parties will freely share all information and does not address the specific issue of contractual structure that may inhibit sharing of information in practice.

Castro-Lacouture and Skibniewski (2006) describe a "business-to-business e-Work system" to foster collaboration between rebar suppliers, construction firms, and design firms. Their work illustrates a system for reducing task durations compared to existing work structures. The B2B e-Work system is implemented using an XML database and ASP. The services architecture in the B2B eWork system involves specified tasks where each participant provides inputs and outputs based on shared information. This system provides mechanisms for tracking changes and allowing the contractors and structural engineers to make concurrent revisions to structural drawings. This mechanism represents a point-based approach to design iteration.

\section{PROCESS MODEL-EZSTROBE}

An EZStrobe $\odot$ (Martinez 1996) model is developed to illustrate the communication flow in the current and proposed future design-construction processes (Figures 1,2). The model deals with the system at a high level using abstraction to represent design options as generic resource tokens. Communication between the components of the EZStrobe (C) model is represented in the control architecture by transmitting these resource tokens. The EZStrobe $\subset$ software is capable of running a discrete event simulation. The results permit a direct comparison of completion times for both the current and proposed processes.

Both EZStrobe (C) models illustrate a communication between a structural engineer (SE), a general contractor (GC), and a rebar fabricator (RF). The RF is assumed to act also as the reinforcing steel detailer. Each of the players is assumed to have the same capabilities in each mod- el. For example, the RF is assumed to staff five detailers in each case. Similarly, the overall design submittal and rebar fabrication process is the same in both models. The difference between the two models is the method of communication between the parties. All communication is linear in the current state. The SE only interacts with the GC; the RF only communicates with the GC. This captures the inefficiencies of a system using point-based design and lacking cross-functional teams.

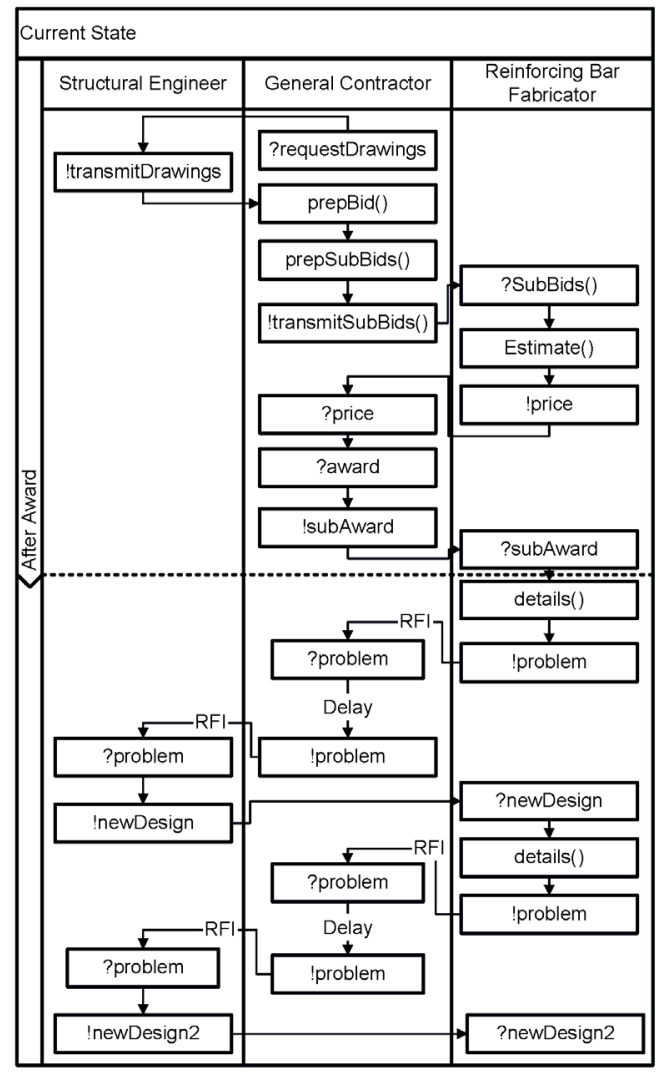

Figure 1: Current state (point-based design)

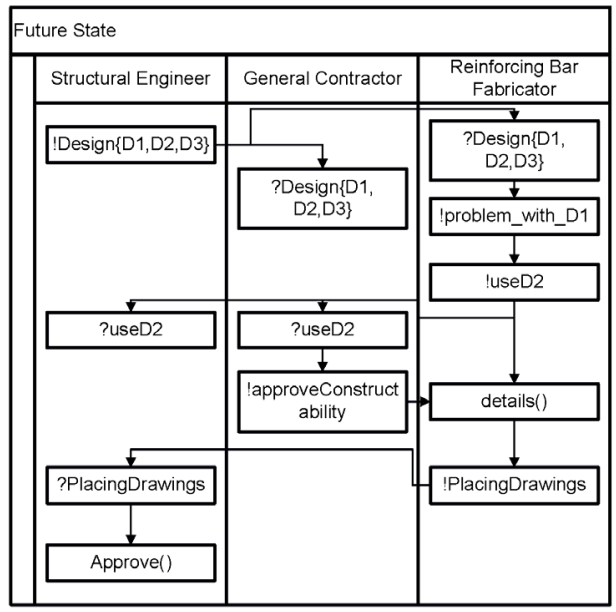

Figure 2: Future state (set-based design) 
Wong, Parrish, Tommelein, and Stojadinovic

\subsection{Activities and Resources in the Current State Model}

The current state of the design industry (point-based design) is modeled in EZStrobe $($ as shown in Figure 3. The activities and resources in the model are:

GC Prepare Bid [Combi Activity] - The General Contractor prepares a bid based on the drawings received from the Structural Engineer. This bid is complete only for the activities which the General Contractor selfperforms. All other activities are completed by subcontractors, so the General Contractor needs to receive prices from the subcontractors before completing a bid.

GC Prepare Subcontractor Bid Package [Normal Activity] - The General Contractor must prepare a description of the work that subcontractors need to complete before bidding out that work.

GC Send Bid Package [Normal Activity] - Bid packages are sent to subcontractors in order to determine pricing information for any work that is performed by subcontractors.

Subcontractor Estimate [Combi Activity] - Estimators in the subcontractors office are responsible for completing a quantity takeoff to determine a quote for the subcontractors work.

Subcontractor's Bid Price is Given to the GC [Normal Activity] - This is the price that is incorporated into the General Contractor's bid for the entire project.

GC Wins the Job [Normal Activity] - At this point, the General Contractor and any subcontractors selected can begin work on the project.

Detail Job [Combi Activity] - A reinforcing steel (rebar) detailer begins to draw out rebar placing drawings, or details, for the project.

RFI Sent to the GC [Combi Activity] - If the rebar detailer is unclear about the Structural Engineer's intentions, an RFI is sent to the General Contractor.

RFI is Passed to the SE [Normal Activity] - The RFI continues through the network of players until it reaches the Structural Engineer, who is able to clarify.

SE Re-designs Part of the Structure [Normal Activity] - The Structural Engineer clarifies the design intent and then the detailing process repeats.

SE Approves the Placing Drawings [Combi Activity] - When detailing is complete, placing drawings are sent to the Structural Engineer to verify their conformance to the original design intent.

Fabricate Rebar for the Job [Normal Activity] Once the rebar placing drawings are approved, the approved details are fabricated and put into storage until they can be shipped to the site (ideally, fabricated pieces are stored for less than twenty-four hours).

Drawings [Queue] - A set of 10 drawings makes up the design of the hypothetical project.
GC Waits [Queue] - for the subcontractors to return price quotes so that the General Contractor's bid can be finished.

Estimator [Queue] - The Reinforcing Steel Fabricator must have an available estimator in order to generate a quote for the General Contractor.

Detailer [Queue] - When the bid is awarded, a detailer must be available to generate details which are used as blueprints for fabrication and placement of rebar.

Good Design [Queue] - In order for the Rebar Fabricator to detail a job, the Structural Engineer's design intent must be clear.

Placing Drawings Sent to the SE [Queue] - The Rebar Fabricator may only fabricate details which are approved by the Structural Engineer, so details are sent to the Structural Engineer as placing drawings.

SE Available to Approve the Placing Drawings [Queue] - Placing drawings tend not to be a top priority for a Structural Engineer working on multiple projects simultaneously, so the approval process is sometimes delayed.

Rebar is Ready to be shipped to the site [Queue] After all approvals are made and the rebar is fabricated, it is stored until the rebar is needed onsite.

\subsection{Activities and Resources in the Future State Model}

The activities and resources which are unique to the future state (set-based) model (Figure 4) are:

RF Reviews the Set of Designs [Combi Activity] In the future state model, there are sets of designs (rather than one single design), all of which are acceptable to the Structural Engineer, which are handed off to the other players for review. The Rebar Fabricator is the first to make a selection as he is the player most aware of rebar constraints (i.e. material availability).

GC Reviews the Fabricator's Design Selection for Constructability [Combi Activity] - The General Contractor reviews the Fabricator's details to determine how easily the details can be incorporated into the schedule and built.

GC Approves the Design [Normal Activity] - The General Contractor must also approve the selected design

A Project Manager Must Be Available to Review the Fabricator's Details [Queue] - Similar to the case of the Structural Engineer's availability to review placing drawings, there must be a Project Manager available to review the constructability of the details. 


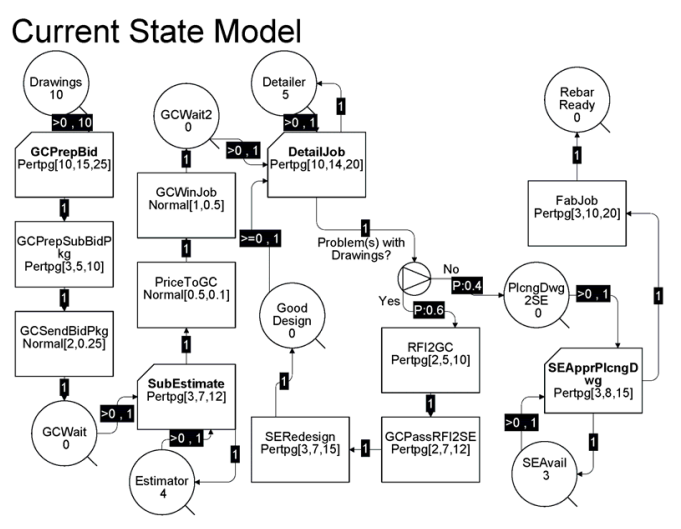

Figure 3: Point-based EZStrobe model

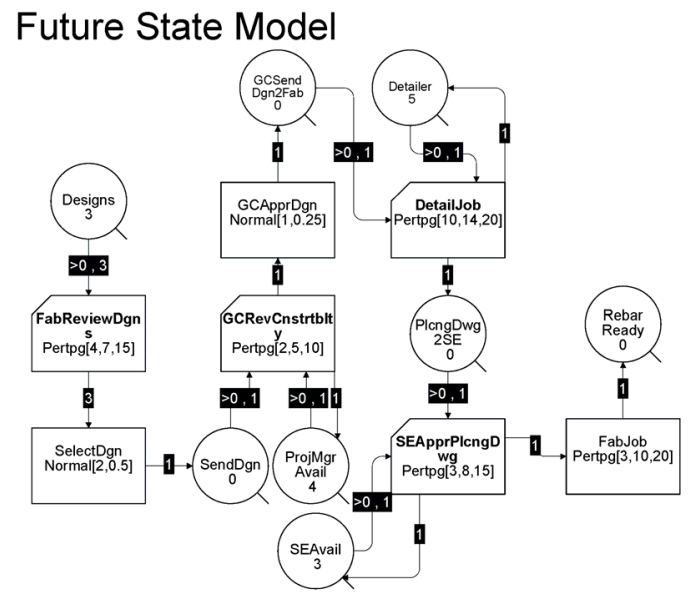

Figure 4: Set-based EZStrobe model

\section{INFORMATION MODEL-WEB SERVICES}

The process models are implemented in Java and developed using the Eclipse IDE and Eclipse Web Tools Platform (e.g., Burnette 2005). The implementation uses Java 5 and Apache Tomcat as the application server on the backend, and PHP 1.5 scripts on Apache httpd 2.0 on the front end. This service-based implementation allows both machine and human interaction with the model implementation. This flexibility allows the implementation to act as a test bed for experimenting with model refinements and simulating a wide range of supply chain dynamics.

The information content in these models is restricted to the parameters needed for expressing the longitudinal reinforcement in a typical reinforced concrete beam.

\subsection{State machines}

Three finite state machine models are developed in order to express the communication required for three distinct entities to work together. While the EZStrobe $(\mathcal{C}$ model uses resource tokens for communication, the Java implementation uses XML as the language for transmitting and storing the required data using HTTP as the communication protocol. One state machine is developed for each of the three project participants: the structural engineer (Figure 5), rebar fabricator (Figure 6) and general contractor (Figure 7). The state machines make explicit the points where information handoffs need to be made and where project details need to be integrated.

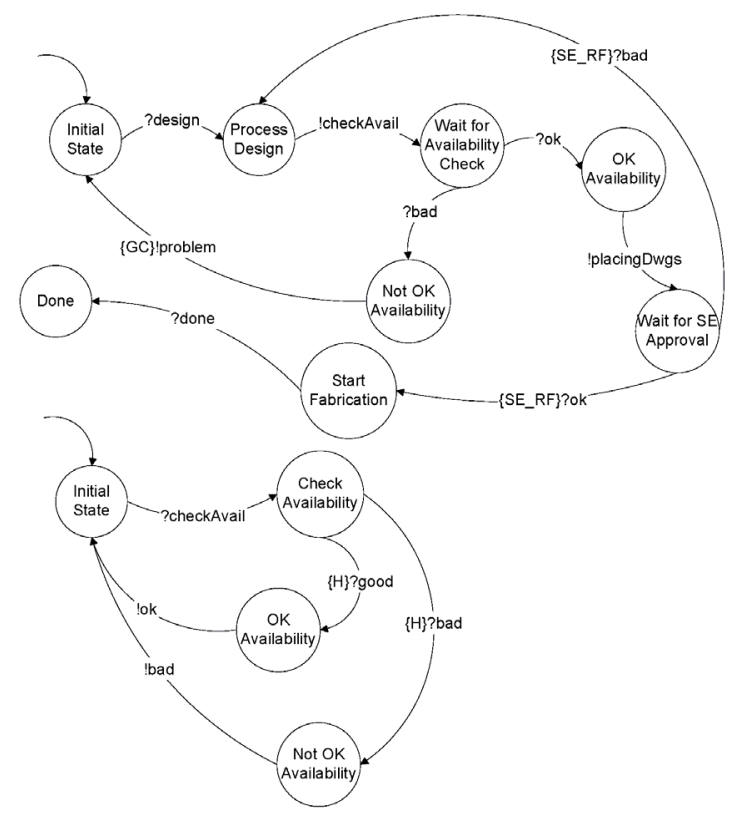

Figure 5: Finite state machine model for SE

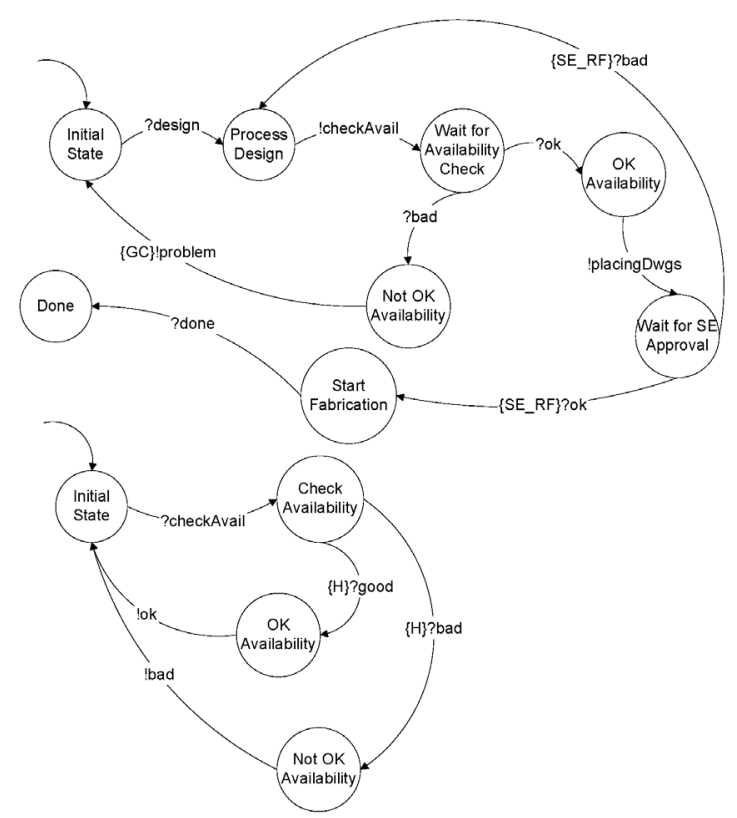

Figure 6: Finite state machine model for RF 


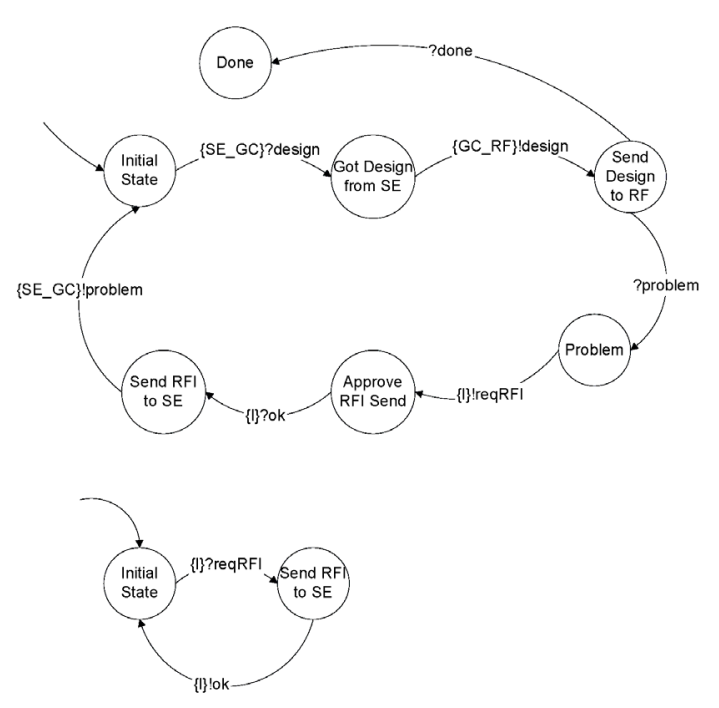

Figure 7: Finite state machine model for GC

\subsection{Database Storage}

A database is used to implement a memory space for variable and state storage in the model. This database stores the state information for the SE, GC, and RF state machines. Apache Derby 10.1 is used as the database implementation for its ease of integration with the Eclipse IDE development tools for web services deployment and local file system storage mechanism. Other databases could be used as long as a JDBC connector is provided by the vendor. The database is initialized using SQL commands that create the necessary tables.

The first database table is created to store the possible options for beam sections. In the model, the SE is the entity responsible for maintaining this data. In the implementation, this data is accessed using the DesignInformationSE web service. This web service is defined using a web services description language (WSDL) file which links to a Java class which contains the code for executing the queries on the database.

The second database table is created for the availability information. In the model, the rebar fabricator is responsible for this information. The data is accessed using the AvailabilityCheckRF web service.

The third database table is used to store the current state of each state machines. This data is accessed using the RoleStates web service which provides methods for getting and setting state.

\subsection{Web Services}

The Java bean web services provide the access to state information and data inside the database tables. The services also provide the implementation mechanism for sending certain signals among the state machine representations. For example, the transmission of problem reports among the project participants has a web service implementation which allows the transmission of a rich problem description as an XML stream. Although this particular functionality is not fully explored in this project, future work can be done to expand on this modeling capability.

Communication that requires only simple information (e.g. integers, floats, short strings) is not implemented as a full web service since doing so would add too much complexity to this particular implementation. However, the framework is in place should future expansion require modification.

The web services were developed first as regular Java classes which provide public methods for accessing the database. Each class contains SQL queries which are executed on the Derby database to read and write information. Once the classes were developed, the Eclipse web tools platform (WTP) was used to automatically generate the corresponding WSDL files which describe how to access the service. The WTP is also used to configure a server for web service deployment.

The DesignInformationSE service provides concrete section parameters and the valid section flag for each enumerated design option. This service retrieves data from the beam design information database table. Each section property has an associated get method which takes one input argument for the unique section id integer. The getProperties method returns all the section properties as a single XML string using the java.util.Properties document type definition as specified in the J2SE API. This XML data could be easily parsed to obtain all of the information in a "chunky" manner as opposed to the "chatty" manner of the individual get methods.

The RoleStates service provides methods for getting and setting the state of each player and the flag indicating whether point-based or set-based workflow is being used. Methods for obtaining the state of the SE, GC, and RF are provided through the getStateSE, getStateGC, and getStateRF methods. Similarly, the setStateSE, setStateGC, and setStateRF methods provide the mechanism for state transitions. The methods setStateSB and getStateSB provide access to the point-based/set-based flag.

Three services, ProblemReportSE, ProblemReportGC, and PlacingDrawingsSE illustrate how the web services can be used to receive information from the various parties. The ProblemReport* services provide a mechanism for receiving input when problems are encountered with a design. The PlacingDrawingsSE provides a mechanism for receiving placing drawings from the rebar fabricator. In this implementation, the recvProblem and recvPlacing methods do not take arguments because the model does not take into account different types of problems and the actual transmission of placing drawings. A more complete model could revise these methods to ac- 
cept string arguments containing XML descriptions of problem reports and placing drawings.

The ResetAll service is provided as a utility for resetting all of the state information in the database to the initial state. This allows all of the information to be reset quickly to restart a model simulation run.

\subsection{Browser-Based User Interface}

A browser-based user interface is developed using PHP which allows for human interaction and visualization of the process. The PHP SOAP module extension is used to make connections to the web services to retrieve state information. Each entity (SE, GC, RF) has an individual page which shows that entity's state as well as any action items that are awaiting input. At each step where the state machine is to transmit a message, the web interface provides a form button for the user to press. The user input is then captured using the HTTP POST method. Each page is set to automatically refresh 10 seconds after the page is fully loaded. This refresh delay models a nominal communication delay between the parties.

The SE page has special information showing a rendering of the concrete design options along with their pertinent section properties and an icon indicating whether they are valid to downstream players (Figure 8). The section rendering is generated just-in-time as a PNG image with the parameters received from the DesignInformationSE web service. The RF page contains information for the current section under consideration for availability as well as readout of the availability information in the database obtained through the AvailabilityCheckRF web service (Figure 9). The GC page does not contain any additional information beyond the state and the pending action (Figure 10).

Because the web pages use a standard browser, the individual users can be located anywhere on the internet. Although the system was deployed on a single machine, because the state information is stored and retrieved via web services, it is possible even for the individual web pages of the different parties to run off different web servers. This implementation scheme allows for easy distribution by leveraging off the network communication provided by the web services. Communication with the web services happens on the server-side using SOAP packets. Future extension could move this communication to the client-side by using asynchronous Javascript and XML (AJAX) techniques.

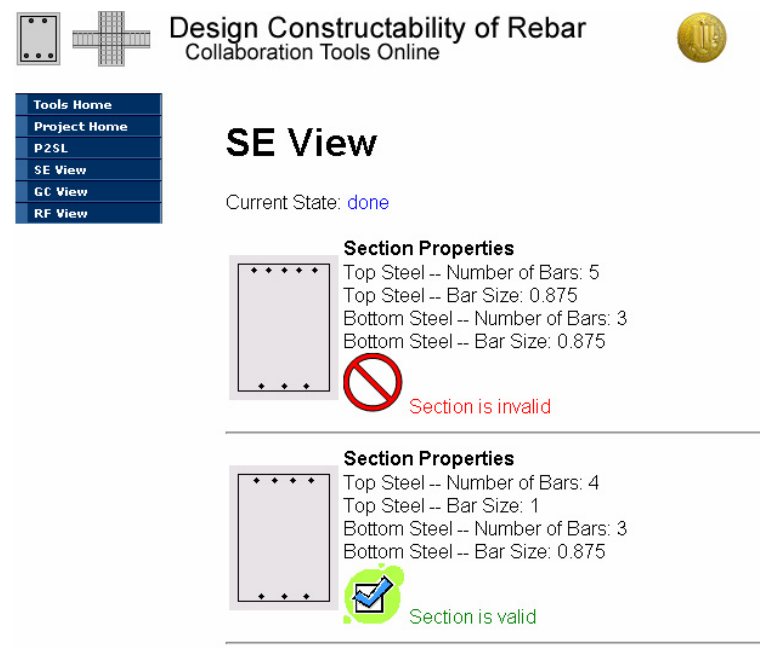

Figure 8: Structural engineer web page view
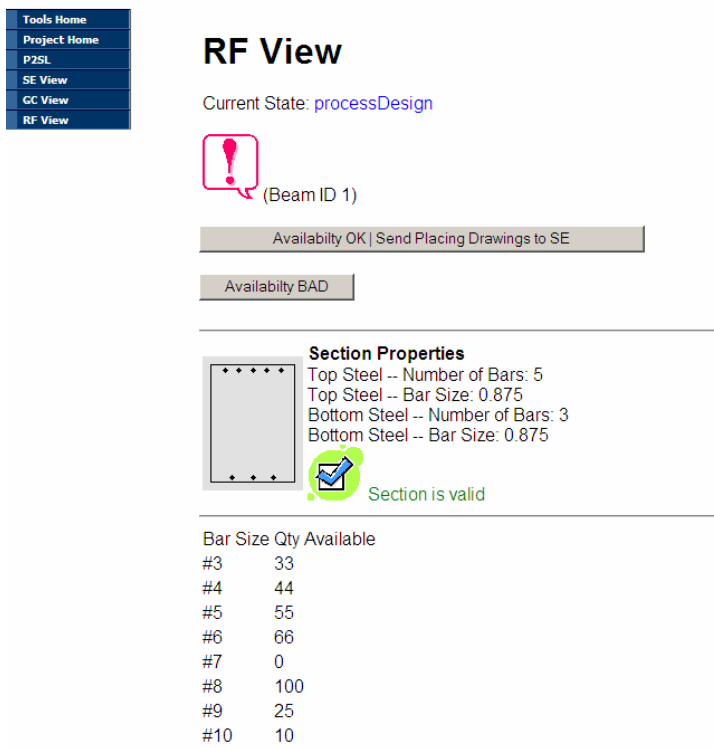

Figure 9: Rebar fabricator web page view

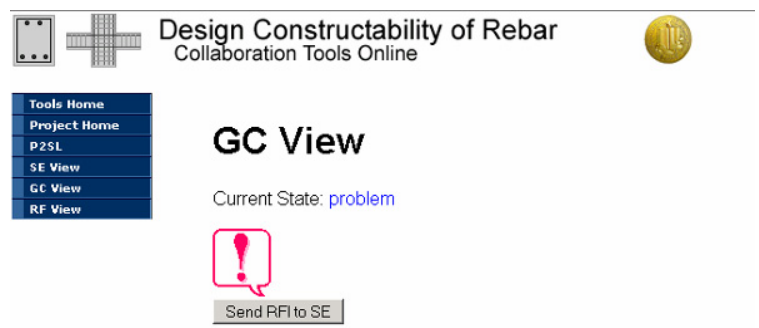

Figure 10: General contractor web page view 


\section{SET-BASED DESIGN SIMULATION}

The activity of selecting longitudinal beam reinforcement in a prototypical reinforced concrete building is used to demonstrate the simulation models and compare set-based design to point-based design. An example design scenario with minimum steel reinforcement areas is pictured in Figure 11. Based on these specifications, the feasible design space can be mapped. Then compatible combinations can be found by intersection after applying hard and soft constraints (Figure 12), which leads to a final design solution that satisfies all the constraints (Figure 13).

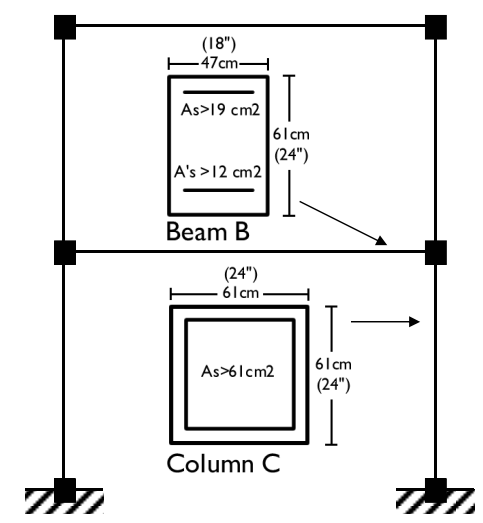

Figure 11: Reinforcing requirements

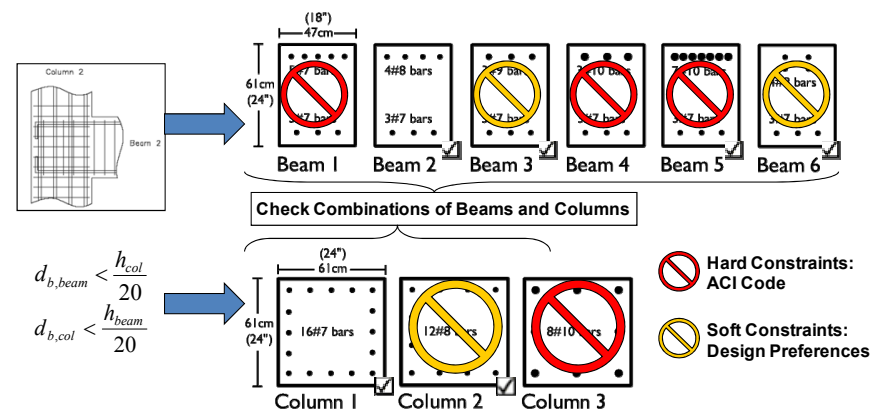

Figure 12: Design set example

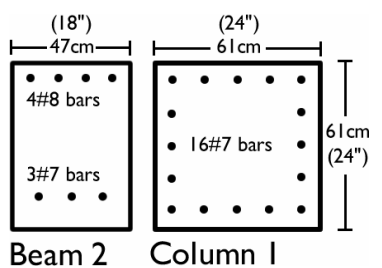

Figure 13: Design solution

Constraints are placed on performing tasks such as generating rebar placing drawings and checking material cost and availability.
The results from the Stroboscope simulation show a completion time reduction of $36 \%$ when using set-based design instead of point-based design. The EZStrobe $($ ) process models were run to test the hypothesis that the future state model would lead to a shorter project completion time. Simulation results show a reduction in completion time from 75 days in the current state to 55 days in the set-based design case. This reduction in time could produce a cost savings based on the decrease in labor time achieved through set-based design.

The XML information flow simulation shows where changes in collaboration structure are required to implement set-based design. The web-based tool illustrates a method of handling the information required to work in a set-based manner.

Running the Java model implementation demonstrates that the amount of additional effort to work in a set-based manner is minimal once the design sets are properly defined. If the design set is defined by the structural engineer, the basic changes in the set-based communication scheme involve only access privileges and a modified work flow. In fact, when problems are encountered, the workflow required for the set-based implementation in the Java model is simpler than the communication for the point-based method. Fewer states are needed in the set-based communication method because bottlenecks with the RFI process through the GC are avoided. If the information required to be passed along can be serialized as XML, then the communication size increases are negligible compared to the potential benefit gained due to minimized delay.

\section{CONCLUSION}

This work is part of a larger project in which the researchers are working with industry practitioners to develop and deploy set-based design methods for rebar design. The simulation models presented here will help deepen understanding on which sets may be developed and which project participants should be involved in pruning sets at which time in the design process. This work also clarifies to what extent information-flow modeling can be used to capture important-though not allprocess features.

\section{ACKNOWLEDGMENTS}

This research was funded by grant CMS-0511044 from the National Science Foundation (NSF) and by a grant from the Concrete Reinforcing Steel Institute (CRSI) whose support is gratefully acknowledged. Any opinions, findings, conclusions, or recommendations expressed in this paper are those of the writers and do not necessarily reflect the views of the NSF or CRSI. 


\section{REFERENCES}

Anwar, N., W. Kanok-Nukulchai, and D. N. Batanov. 2005. "Component-based information oriented structural engineering applications". ASCE, J. of Computing in Civil Engineering 19(1).

Ballard, G. 2000. "Positive vs. Negative Iteration in Design." Proc. 8th Ann. Conf. of the Int'l. Group for Lean Construction (IGLC-8), Univ. of Sussex, Brighton, UK, 44-55.

Burnette, E. 2005. Eclipse IDE Pocket Guide. Sebastopol, CA: O'Reilly.

Castro-Lacouture, D. and M. Skibniewski. 2006. "Implementing a B2B e-Work System to the Approval Process of Rebar Design and Estimation". ASCE, $J$. of Computing in Civil Engineering 20(1).

Pena-Mora, F. and K. Choudri. 2001. "Web-centric framework for secure and legally binding electronic transactions". ASCE, J. of Computing in Civil Engineering 15(4).

Pena-Mora, F. and C.-Y. Wang. 1998. "Computer Supported Collaborative Negotiation Methodology". ASCE, J. of Computing in Civil Engineering 12(2).

Sobek II, D.K., A. Ward, and J. K. Liker. 1999. "Toyota's Principles of Set-Based Concurrent Engineering." Sloan Management Review 40(2):67-83.

Ward, A., J.K. Liker, J.J. Cristiano, and D. K. Sobek II. 1995. "The Second Toyota Paradox: How Delaying Decisions Can Make Better Cars Faster." Sloan Management Review 36(3):43-61.

\section{AUTHOR BIOGRAPHIES}

JOHN-MICHAEL WONG is a graduate student in Civil and Environmental Engineering at the University of California, Berkeley. His research interest is in performancebased design and evaluation of structures, with a focus on post-disaster assessment, data storage mechanisms for structural performance data, and computer tools for structural engineering applications. His webpage can be found via <www . jmwong. com>.

KRISTEN PARRISH is a graduate student in Civil and Environmental Engineering at the University of California, Berkeley. Her research interests are the engineering design processes, especially structural design. More specifically, she is interested in set-based design as it relates to reinforced concrete. Her webpage can be found via <www. ce.berkeley.edu/ parrishk/interest . htm $>$.

IRIS D. TOMMELEIN is Professor of Engineering and Project Management in the Department of Civil and Environmental Engineering at University of California, Berkeley. She directs, jointly with Glenn Ballard, the Project
Production Systems Laboratory. Her research interest is in developing principles of production management for project delivery in architecture-engineering-construction industry, what is termed 'lean construction.' Iris has strong analytical, computational, and writing skills. She has a proven research track record that includes developing industry case studies. Her work often involves computeraided design, planning, scheduling, simulation, mapping, and visualization of construction processes. She is a member of the American Society of Civil Engineers, a member of the International Group for Lean Construction, and she serves on the Board of Directors of the Lean Construction Institute. You can reach her by email at tommelein@ce.berkeley.edu and her web address is <www.ce.berkeley.edu/ tommelein>.

BOZIDAR STOJADINOVIC is an Associate Professor with the Department of Civil and Environmental Engineering at the University of California, Berkeley. His primary research interest is in probabilistic performancebased seismic design of civil structures. In this area, he specializes in performance-based evaluation of highway bridges and nuclear facility structures using the methodology developed within the Pacific Earthquake Engineering Center at Berkeley. In the area of application of information technologies in structural engineering, he is developing new experimental methods and, in particular, the hybrid simulation method for evaluation of civil structures using hybrid models that combine physical and numerical substructures. He is a key member of the nees@berkeley Equipment Site team and a leader in developing methods for geographically distributed hybrid simulation using NEES facilities and methods for evaluating the quality of hybrid simulations. He is, also, involved in using innovative sensor and communication technologies to instrument structures and interpret the state of the structure after an extreme event given a large number of unreliable sensor readings. His web address is $<$ ww. ce. berkeley. edu/ boza $>$. 